\title{
Brucella infection at cardiac pacemaker site in a patient who had consumed raw caribou meat in Northern Canada
}

\author{
Jamil N. Kanji MD, Lynora Saxinger MD
}

Cite as: CMAJ 2018 September 17;190:E1108-10. doi: 10.1503/cmaj.170234

A n 82-year-old man was transferred from Inuvik (Northwest Territories, Canada) to a tertiary hospital in Alberta for evaluation of an infection at a permanent pacemaker site. The pacemaker had been inserted in 2005 to treat third-degree heart block. The patient had experienced increasing discomfort, erythema and warmth at the site of the pacemaker generator for the preceding year, with intermittent fevers and sweats. His medical history was otherwise unremarkable.

Blood cultures ordered in Inuvik were negative and the patient received treatment with intravenous cefazolin for cellulitis with no improvement. At the tertiary hospital, physical examination showed erythema without drainage at the pacemaker pocket, no heart murmurs and no evidence of peripheral embolic phenomena. A transesophageal echocardiogram showed no valvular vegetations. Given the prolonged recurring fevers and signs suggestive of infection at the generator site, cardiologists at the tertiary hospital decided to remove the pacemaker via laser lead extraction and place a temporary central venous pacemaker. No other foci of infection were identified. The patient received perioperative treatment with cefazolin and empiric intravenous vancomycin after the procedure. Two separate cultures of the extracted ventricular leads and ventricular tissue, respectively, were positive for gram-negative bacilli. Ciprofloxacin was added and infectious diseases consulted. The laboratory was notified that the differential diagnosis included brucellosis, given the patient's setting and history.

When the organism was identified presumptively as Brucella species, treatment was switched to intravenous gentamicin and oral doxycycline. Given the bacterial growth on the sample of endovascular ventricular tissue despite negative blood cultures, we made a clinical diagnosis of endocarditis. Blood culture sensitivity for Brucella can be poor, which means that the Duke Criteria for infective endocarditis may not be reliable when Brucella is the infecting organism. ${ }^{1}$

The bacterial isolate was confirmed as Brucella suis (biotype 4) by the National Microbiology Laboratory (Winnipeg). The organism was susceptible to doxycycline (minimum inhibitory

\section{KEY POINTS}

- Hunting, butchering and consuming of uncooked caribou meat as part of a traditional diet is common in Northern Canada, and Canadian physicians should be aware of the potential for Brucella suis zoonosis.

- Brucella species zoonoses, which may also occur after consumption of unpasteurized dairy products, present as either acute or long-term infection; focal infection, for example, infection of implantable devices, is common.

- If physicians suspect infection with Brucella species, the microbiology laboratory should be notified to ensure specimens are processed appropriately to reduce risk of infection in laboratory workers.

concentration (MIC) of less than $0.03 \mu \mathrm{g} / \mathrm{mL}$ ) and gentamicin (MIC $2 \mu \mathrm{g} / \mathrm{mL}$ ) by standardized breakpoint interpretation, with both ciprofloxacin and rifampin having MICs of $0.25 \mu \mathrm{g} / \mathrm{mL}$.

On detailed questioning after we identified the bacterial cause of infection, our patient stated that he had hunted and skinned caribou in several areas of the Northwest Territories and had ingested uncooked caribou meat and blood. He and his family were considered subsistence hunters, relying on store-bought groceries only to supplement food they secured by hunting and fishing. Moreover, he told us that his brother (with whom he frequently hunted), had been treated previously for Rangiferene brucellosis (zoonotic transmission of Brucella suis biotype 4).

The patient completed seven days of treatment with gentamicin and doxycycline in hospital. Because he was pacemaker dependent, a new permanent pacemaker was inserted (on the opposite side from the original). He was transferred home to continue therapy with oral doxycycline and rifampin for 12 weeks. Follow-up blood cultures after 12 weeks of therapy were negative.

At four years of follow-up with the infectious diseases department, the patient was doing well with no adverse effects, systemic symptoms, changes at the site of the new pacemaker or clinical signs of relapse of infection. 


\section{Discussion}

Brucellosis is a zoonosis caused by a gram-negative bacillus, of which four species are most implicated in human disease (i.e., Brucella abortus, Brucella canis, Brucella melitensis and B. suis). It is prevalent worldwide. The organism may be concentrated in the urine, milk, tissues and products of conception (e.g., placenta) of infected animals, and can be transmitted through ingestion, direct contact and aerosol inhalation. People in direct contact with infected animal materials in settings such as stables, abattoirs and laboratories, and those involved in activities such as assisting birth and butchering, are at high risk. Consumption of unpasteurized dairy products made using the milk of infected animals is an important route of spread globally, especially in the Middle East. ${ }^{1}$ Brucella canis in dogs imported from abroad also poses a possible risk, particularly to animal health workers. ${ }^{2}$ In one case series of infections caused by Brucella species in abattoir workers, the primary identified routes of $B$. suis transmission were cutaneous exposure, inhalational via aerosol (during skinning and slaughter) and ingestion of undercooked meat. ${ }^{3}$ Human-to-human transmission of brucellosis is uncommon but has been reported to occur through mother-to-child (vertical) transmission, breastfeeding, sexual intercourse, blood transfusion, bone marrow transplantation and occupationally through exposure to infected birth products or laboratory cultures. ${ }^{4}$

The course of brucellosis in humans can be acute, subacute or chronic. Most patients present with unexplained fevers lasting days to weeks. Focal infection most commonly manifests as spondylodiscitis (via hematogenous spread), but involvement of the genitourinary, pulmonary, ocular and gastrointestinal systems can occur. ${ }^{1}$ Laboratory-confirmed brucellosis is reportable to local public health authorities across all Canadian jurisdictions. About 10 cases of human brucellosis are reported per year in Canada reflecting an incidence of less than 1 per 100000 population. Alberta averages one case per year and Ontario averages four (2003-2012). ${ }^{2,5}$ In one review of 19 cases of brucellosis in Ontario, travel outside Canada was the main risk factor for more than $75 \%$ of the cases, with consumption of unpasteurized dairy products reported in 13 of 19 cases. $^{2}$ Regions of higher risk include the Middle East, Mediterranean and Mexico.

As of 2016, the Canadian Food Inspection Agency has indicated that Canada's eradication program for bovine brucellosis in livestock was successful, with no cases in cattle since 1989. ${ }^{2}$ Brucella infection of pigs, goats and sheep has not been reported in livestock in Canada. ${ }^{2}$

Zoonotic transmission of $B$. suis biotype 4 has been documented in Canada's Inuit communities of the Northwest Territories and Baffin Island, with the primary reservoir being caribou (Rangifer tarandus). ${ }^{6} \mathrm{~A}$ considerable proportion of Inuit communities across the Arctic subsist mainly by hunting, fishing and gathering, ${ }^{7}$ with some groups unable to maintain an adequate diet without hunting caribou. ${ }^{8}$ Inuit hunters often eat some of the raw meat when dressing hunted animals, which puts them at risk of zoonotic infection. ${ }^{6}$ Canadian physicians should be aware that uncooked caribou meat is a nutritional need and a local delicacy in many northern communities, representing an ongoing risk of acquiring Rangiferene brucellosis. ${ }^{8}$ Therefore, brucellosis should be considered a potential diagnosis in febrile individuals returning from endemic areas with a history of ingestion of unpasteurized dairy products or exposure to butchering and eating infected caribou in the far North.

If brucellosis is suspected, blood should be collected for culture (yield of $15 \%-70 \%$ ). ${ }^{1}$ Culture of other body fluids or tissue from suspected areas of involvement is an important source of microbiological growth when results from blood cultures are negative (as in our patient). Importantly, culture allows speciation and determinations of antimicrobial susceptibilities. The laboratory should be notified whenever Brucella is suspected as a cause of infection to ensure that appropriate laboratory precautions are taken. Brucella is a level 3 pathogen and laboratory personnel are at risk of infection. ${ }^{5}$

Serologic diagnosis (total antibodies to $B$. abortus, B. suis and $B$. melitensis) may also be helpful. In general, a titre of more than 1:160 or a fourfold increase between acute and convalescent specimens is considered positive, with a sensitivity and specificity of $64.7 \%-84.6 \%$ and $99.5 \%$, respectively. ${ }^{1}$ Polymerase chain reaction and other molecular tests to detect various Brucella species may be useful in specific cases.

First-line antimicrobial treatment for brucellosis is doxycycline in combination with an aminoglycoside or rifampin, depending on the site of the infection. Streptomycin was classically the aminoglycoside of choice, but gentamicin is a reasonable alternative given the ease of availability and proven equivalent efficacy. ${ }^{9}$ Other agents that may be effective include fluoroquinolones and co-trimoxazole. From a laboratory perspective, Clinical and Laboratory Standards Institute interpretations for susceptibility are available only for gentamicin, streptomycin, doxycycline, tetracycline and co-trimoxazole, with no standardized breakpoints to interpret susceptibility to fluoroquinolones or cephalosporins. Given these complexities, consultation with a specialist in infectious diseases is advised to assist in the treatment of brucellosis. References with further information about brucellosis can be found in Appendix 1, available at www.cmaj.ca/lookup/suppl/ doi:10.1503/cmaj.170234/-/DC1.

In our patient's case, management consisted of surgical removal of the permanent pacemaker and its leads, along with prolonged antibiotic therapy for systemic, presumed endovascular brucellosis. Although initial blood culture results were negative, samples from both the device and ventricular tissue were culture positive. Recommended therapy for endovascular infection includes a combination of an aminoglycoside, doxycycline and rifampin, with at least one week of treatment with an aminoglycoside (as in our patient) to prevent relapse and reduce mortality. ${ }^{9,10}$ A recent case report described that the duration of treatment in patients with endocarditis related to an infection caused by $B$. melitensis in a cardiovascular implantable electronic device-ranged from three to six months. ${ }^{11}$ Antibiotic therapy alone resulted in relapse because of persistently infected devices. ${ }^{11}$ Our patient received 12 weeks of therapy after surgical replacement of his pacemaker, with no evidence of relapse four years after infection. 


\section{References}

1. Franco MP, Mulder M, Gilman RH, et al. Human brucellosis. Lancet Infect Dis 2007;7:775-86

2. Monthly infectious diseases surveillance report. Toronto: Public Health Ontario; May 2013. Vol 2. Issue 5. Available: www.publichealthontario.ca/en/ DataAndAnalytics/Documents/2013_May_PHO_Monthly_Report.pdf (accessed 2018 Feb. 5).

3. Buchanan TM, Hendricks SL, Patton CM, et al. Brucellosis in the United States, 1960-1972; An abattoir-associated disease. Part III. Epidemiology and evidence for acquired immunity. Medicine (Baltimore) 1974;53:427-39.

4. Tuon FF, Gondolfo RB, Cerchiari N. Human-to-human transmission of Brucella a systematic review. Trop Med Int Health 2017;22:539-46.

5. Public health disease management guidelines: Brucellosis. Edmonton: Alberta Government; 2012. Available: https://open.alberta.ca/publications/ brucellosis\#summary (accessed 2018 Apr. 8).
6. Forbes LB. Isolates of Brucella suis biovar 4 from animals and humans in Canada, 1982-1990. Can Vet J 1991;32:686-8.

7. Nancarrow TL, Chan HM. Observations of environmental changes and potential dietary impacts in two communities in Nunavut, Canada. Rural Remote Health 2010;10:1370.

8. Chiu A, Goddard E, Parlee B. Caribou consumption in northern Canadian communities. J Toxicol Environ Health A 2016;79:762-97.

9. Ariza J, Bosilkovski M, Cascio A, et al. Perspectives for the treatment of brucellosis in the 21st century: the lonnina recommendations. PLoS Med 2007;4:e317.

10. Koruk ST, Erdem H, Koruk I, et al. Management of Brucella endocarditis: results of the Gulhane study. Int J Antimicrob Agents 2012;40:145-50.

11. Al-Adsani W, Ahmad A, Al-Mousa M. A case of Brucella melitensis endocarditis in a patient with cardiovascular implantable electronic device. Infect Drug Resist 2018;11:387-90.

\section{Competing interests: None declared.}

This article has been peer reviewed.

The authors have obtained patient consent.

Affiliations: Division of Infectious Diseases, Department of Medicine (Kanji, Saxinger), University of Alberta; Division of Medical Microbiology, Department of Medical Microbiology \& Immunology (Kanji, Saxinger), University of Alberta; Provincial Laboratory for Public Health (Microbiology) (Kanji), University of Alberta Hospital, Edmonton, Alta.
Contributors: Both of the authors contributed equally to the conception, drafting and critical revision of the manuscript for important intellectual content; gave final approval of the version to be published; and agreed to be accountable for all aspects of the work.

Acknowledgements: The authors thank Dr. Leah Seaman for her review of this manuscript and for her northern medical expertise and management.

Correspondence to: Jamil Kanji, jamil.kanji@ahs.ca 